\title{
A Sustained Response of Maintenance Therapy in Pancreatic Acinar Cell Carcinoma (PACC): A Case Report and Literature Review
}

\author{
Shahid Gilani* and Apurna Jegannathen \\ University Hospitals of North Midlands NHS Trust, United Kingdom \\ *Corresponding Author: Shahid Gilani, University Hospitals of North Midlands \\ NHS Trust, United Kingdom.
}

Received: August 10, 2020

Published: August 24, 2020

(C) All rights are reserved by Shahid Gilani

and Apurna Jegannathen.

\begin{abstract}
We describe a 50 year old male patient who was histologically diagnosed to have acinar cell tumour of pancreas. He initially presented with localized pancreatic cancer. He underwent distal pancreatectomy with splenectomy for pT3pNxpMxR1 disease, confirming acinar cell tumour. He was started on adjuvant chemotherapy with Gemcitabine but was discontinued after 2 cycles due to anaphylaxis. On disease relapse, Oxaliplatin plus Capecitabine was started but stopped after 5 cycles because of disease progression. But surprisingly a later CT scans after 3 months showed disease response which continued for almost 6 months without any treatment. We think this was an unusual late response. But unfortunately his disease progressed again shortly after this. At that time we re-challenged him with the same chemotherapy. More than four years from his initial diagnosis, this patient on maintenance treatment showed further clinical and radiological improvement in his disease.
\end{abstract}

Keywords: Maintenance Therapy; Pancreatic Acinar Cell Carcinoma (PACC); Gemcitabine; Oxaliplatin; Capecitabine

\section{Introduction}

Each year 8463 new cases of pancreatic cancer are diagnosed in UK [4-7]. Incidence of pancreatic cancer has remained the same over last two decades in UK affecting equal proportions of men and women accounting for 3\% of total new cancers [4-7]. Due to longevity of population incidence of pancreatic cancer is going to increase in future [8]. Pancreatic tumours can arise from either endocrine or exocrine part of pancreas [8]. Almost 95\% of these cancers are exocrine in origin arising from ductal part [4-7]. Pancreatic acinar cell carcinoma (PACC) is rare tumour comprising of $1-2 \%$ of exocrine pancreatic cancers $[8,9]$. PACC is genomically distinct from other pancreatic cancers [17].

\section{Background}

Acinar cell carcinoma is a rare form of pancreatic malignancy comprising of only $1-2 \%$ of all pancreatic cancers [1]. They tend to occur more in men at the age of seventy [2]. Presenting symptoms are usually non-specific. Sometimes it is associated with increased serum level of lipase [3]. We report a young man who presented with general ill health and weight loss at the age of fifty years. His disease not only responded well to initial chemotherapy but also remained stable on maintenance chemotherapy. He lived for 48 months after his initial diagnosis.

\section{Case Report}

A 50 year old male presented with weight loss and general tiredness. He had no relevant past medical history or family history. His WHO performance status was zero. CT scans picked up a left hypochondrial mass which later confirmed histologically as adenocarcinoma. He underwent splenectomy and distal pancreatectomy. Final histology came back as well differentiated acinar cell carcinoma of pancreas (pT3 pNx pMx R1). He was started on 
adjuvant chemotherapy with Gemcitabine but discontinued due to anaphylactic reaction. Three months later a surveillance CT scans raised suspicion of local recurrence and possible liver metastases. These findings were confirmed by PET CT scan (Figure 1). Therefore, Oxaliplatin plus Capecitabine regimen was started in palliative settings. After 5 cycles his disease seemed progressing in the liver (Figure 2). He declined participating in clinical trial or to have further chemotherapy treatment. Three months later he presented with abdominal pain. Surprisingly CT scan at that time showed shrinkage of pancreatic tumour with disappearance of the liver lesions (Figure 3). This phenomenon may be a manifestation of late response to previous chemotherapy. But unfortunately his disease progressed again six months later which was confirmed by CT and MRI scans. A decision to re-challenge with Oxaliplatin plus Capecitabine was taken. After 4 more cycles, a remarkable response was seen again (Figure 4). We had to stop Oxaliplatin due to neurotoxicity but we continued maintenance single agent Capecitabine. Further imaging showed persistent response with no further progression even after 48 months from his initial diagnosis. This patient lived on maintenance treatment with good performance status for four years from his original diagnosis.

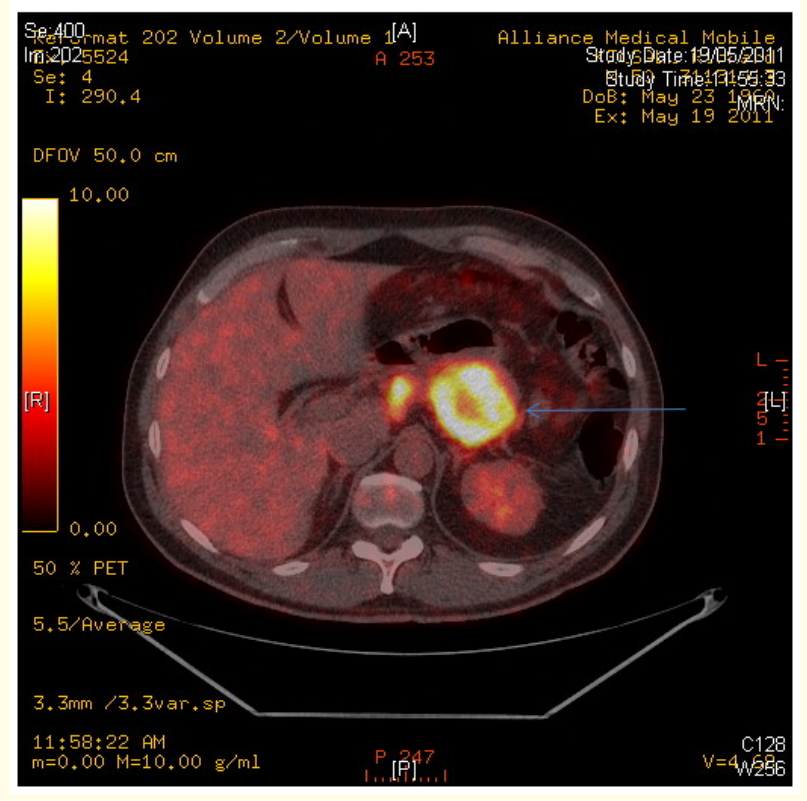

Figure 1: PET CT Scan showing pancreatic cancer recurrence.

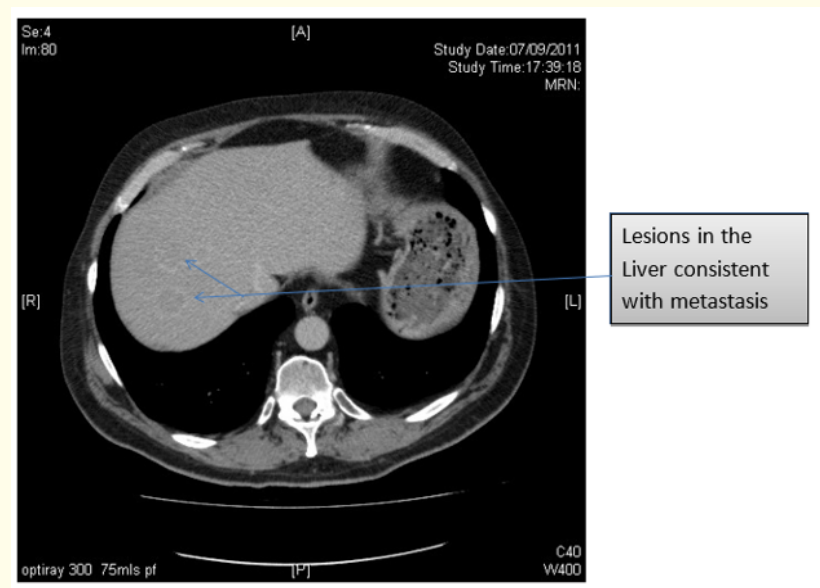

Figure 2: CT scan: Showing disease progression in the liver.

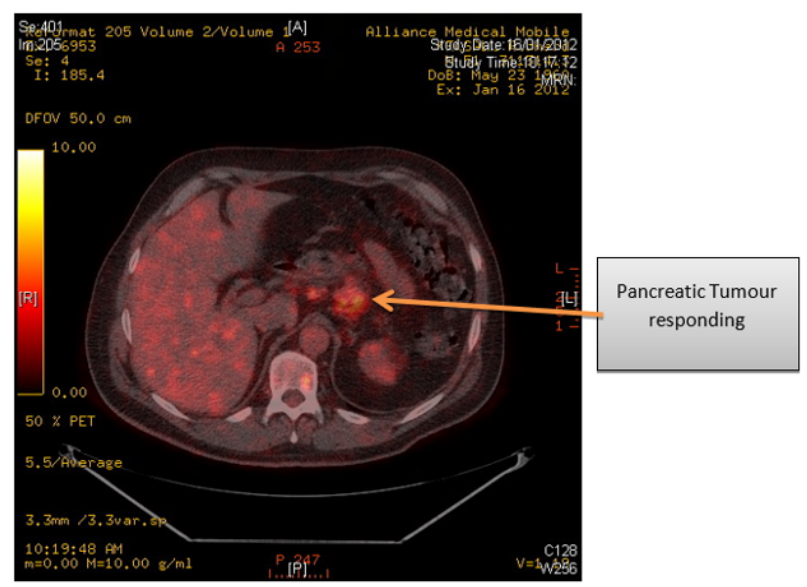

Figure 3: CT scan: Showing late response after 3 months of stopping treatment.

There was continued disease response with maintenance capecitabine treatment. He was being monitored with regular surveillance CT scans until his disease progressed.

\section{Discussion}

Acinar carcinoma is more prevalent in males as compared to females with mean age between 60 to 70 years [3]. It commonly 


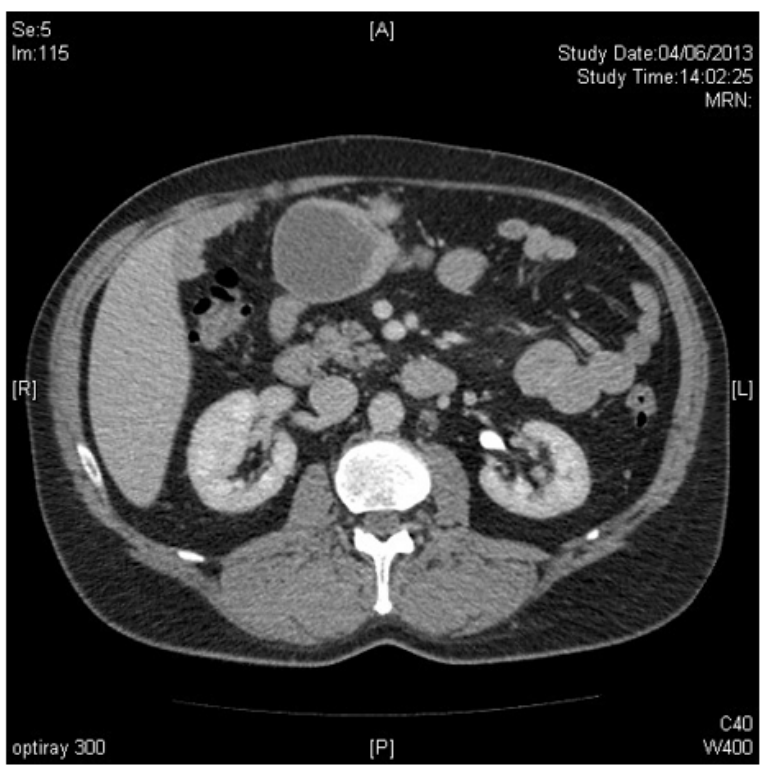

Figure 4: CT scan: Pancreatic tumour still responding and liver remains clear.

presents with weight loss, nausea and abdominal discomfort [3]. Serum lipase level may be elevated in some patients manifesting Schmid's triad comprising of polyarthritis, eosinophilia and fat necrosis [10]. Rarely acinar cell carcinoma can also present with endocrine abnormalities [3]. Our case is unique in this sense that patient is relatively young although he presented with symptoms known to occur in this type of cancer i.e. weight loss and constitutional symptoms.

Most pancreatic cancers have poor prognosis [11]. Mean survival remains 4 - 8 months for advanced pancreatic cancers [12]. No separate protocols exist to treat acinar cell tumours. At present this rare type malignancy is being treated exactly the same way as other types of exocrine pancreatic tumours. Gemcitabine remained the mainstay of treatment for advanced pancreatic cancer (APC) for many years [1]. Unfortunately, many earlier trials have failed to show survival benefits who tried to add different chemotherapeutic agents onto gemcitabine $[9,12]$. But later on two meta-analyses revealed that combining gemcitabine either with capecitabine [13] or oxaliplatin [14] has resulted in lengthening overall survival slightly. Recently a combination triplet regimen
(Gemcitabine, Oxaliplatin and Capecitabine) has been tried with some benefits in APC increasing median time to progression of 4.3 months [15]. But this comes with a price of toxicity and rapid development of resistance.

A similar case of acinar cell carcinoma of pancreas was reported by Monique Antoine., et al. in 2007 who survived for 37 months after treatment with multiple chemotherapeutic agents [16]. But our case is unique in this respect that he survived even longer after receiving only single regimen of oxaliplatin plus capecitabine followed by maintenance chemotherapy with capecitabine alone.

\section{Conclusion}

Oxaliplatin plus Capecitabine combination may have a role to play in the treatment of acinar cell pancreatic cancer. Late manifestation of response may occur and long term disease stabilization may be possible with maintenance chemotherapy in some patients. This may be due to underlying biological and genetic factors which need to be explored further by genomic profiling. For example, fusions in RAF genes and mutually exclusive inactivation of DNA repair genes represent novel potential therapeutic targets that are altered in over two thirds of these tumors [17].

\section{Competing Interests}

None.

\section{Patient Consent}

Obtained.

\section{Bibliography}

1. Solcia E., et al. "Tumors of the pancreas". In: Atlas of Tumor Pathology; $3^{\text {rd }}$ Series, fascicle 20. Washington, DC, USA: AFIP, Armed Forces Institute of Pathology (1997): 103-114.

2. Handley ER., et al. "Pancreatic acinar cell carcinoma. Brigham RAD Case Lists". Harvard Medical School, Department of Radiology, Brigham and Women's Hospital (2006).

3. Aqel B., et al. "Recurrent pancreatitis due to a cystic pancreatic tumor: a rare presentation of acinar cell carcinoma". Journal of Pancreas 5 (2004): 151-154.

4. Data were provided by the Office for National Statistics on request (2012). 
5. Data were provided by ISD Scotland on request (2012).

6. Data were provided by the Welsh Cancer Intelligence and Surveillance Unit on request (2012).

7. Data were provided by the Northern Ireland Cancer Registry on request (2012).

8. Raimondi S., et al. "Epidemiology of pancreatic cancer: an overview". Nature Reviews Gastroenterology and Hepatology 6 (2009): 699-708.

9. Sultana A., et al. "Meta-analyses of chemotherapy for locally advanced and metastatic pancreatic cancer". Journal of Clinical Oncology 25 (2007): 2607-2615.

10. Ashley SW and Lauwers GY. "Case records of the Massachusetts General Hospital. Weekly clinicopathological exercises. Case 37-2002. A 69-year old man with painful cutaneous nodules, elevated lipase levels, and abnormal results on abdominal scanning". The New England Journal of Medicine 347 (2002): 1783-1791.

11. Klimstra DS., et al. "Acinar cell carcinoma of the pancreas. A clinicopathological study of 28 cases". The American Journal of Surgical Pathology 16 (1992): 815-837.

12. Yip D., et al. "Chemotherapy and radiotherapy for inoperable advanced pancreatic cancer". The Cochrane Database of Systematic Reviews 3 (2006): CD00209.

13. Cunningham D., et al. "Phase III randomized comparison of gemcitabine versus gemcitabine plus capecitabine in patients with advanced pancreatic cancer". Journal of Clinical Oncology 27 (2009): 5513-5518.

14. Heinemann V., et al. "Meta-analysis of randomized trials: evaluation of benefit from gemcitabine-based combination chemotherapy applied in advanced pancreatic cancer". BMC Cancer 8 (2008): 82.

15. V Hess., et al. "Combining gemcitabine, oxaliplatin and capecitabine (GEMOXEL) for patients with advanced pancreatic carcinoma (APC): a phase I/II trial". Annals of Oncology 21 (2010): 2390-2395.
16. Monique Antoine., et al. "Long-Term Survival in a Patient with Acinar Cell Carcinoma of Pancreas. A Case Report and Review of Literature”. Pancreas Journals 8.6 (2007): 783-789.

17. Vhmielecki J., et al. "Comprehensive genomic profiling of pancreatic acinar cell carcinomas identifies recurrent RAF fusions and frequent inactivation of DNA repair genes". Cancer Discovery 4.12 (2014): 1398-1405.

\section{Assets from publication with us}

- Prompt Acknowledgement after receiving the article

- Thorough Double blinded peer review

- Rapid Publication

- Issue of Publication Certificate

- High visibility of your Published work

Website: www.actascientific.com/

Submit Article: www.actascientific.com/submission.php Email us: editor@actascientific.com

Contact us: +919182824667 